\title{
SUSCETIBILIDADE BIOLÓGICA DO TRIATOMA SORDIDA E TRIATOMA INFESTANS A DELTAMETRINA E LAMBDACYHALOTRINA EM CONDIÇÕES DE CAMPO
}

\author{
Liléia Diotaiuti e Claúdio Texeira Pinto
}

\begin{abstract}
No município de Posse-Goiás, foram constituídos três grupos homogêneos de casas borrificadas com deltamethrin $5 \%$ - $25 \mathrm{mg}$ i.a. $/ \mathrm{m}^{2}$ nas formulações pó molhável e suspensão concentrada, ou lambdacyhalothrin $10 \%-30 \mathrm{mg}$ i.a. $/ \mathrm{m}^{2}$ pó molhável, para estudo da suscetibilidade biológica do Triatoma sordida e do Triatoma infestans até 360 dias após a borrifação. Dez ninfas de terceiro estádio de cada espécie foram colocadas, por casa, em contato com as paredes por 72 horas, em cones plásticos desenvolvidos pela OMS para testes com mosquitos adultos. Trinta dias após a exposição, anotava-se o número de insetos mortos. Estes testes foram realizados aos 90,270 e 360 dias após a borrifação das casas. Os resultados revelaram persistência da ação inseticida dos três produtos até a última avaliação realizada, e a igual suscetibilidade do Triatoma sordida e do Triatoma infestans a todas as formulações.
\end{abstract}

Palavras chaves: Suscetibilidade. Triatoma sordida. Triatoma infestans. Deltametrina. Lambdacyhalotrina.

O programa de Controle da Doença de Chagas/SUCAM-Ministério da Saúde tem, nos últimos anos, utilizado piretróides para o combate a triatomíneos. Os resultados obtidos indicam superioridade destes inseticidas ao hexacloro benzeno (BHC), já que protegem a casa por maior tempo, apresentam al to poder Knock-down, baixa toxicidade para os homens e animais domésticos ${ }^{2}$, não deixam resíduo nas paredes e não têm cheiro, o que faz com que sejam muito bem aceitos pela população.

O tratamento domiciliar com estes inseticidas leva à redução ou desaparecimento imediato da população triatomínica, permanecendo os intradomicílios negativos por períodos superiores a nove meses ${ }^{3}$. Devido a sua mais

\footnotetext{
Departamento de Parasitologia, Instituto de Ciências Biológicas da Universidade Federal de Minas Gerais. Belo Horizonte, MG.

Quimio Produtos Químicos Comércio e Indústria SA. Rio de Janeiro, RJ.

Enderefo para correspondência: Dra Liléia Diolajuti. Depto. de Parasitologia/ICB/UFMG. Caixa Postal 2486, 31270 Belo Horizonte, MG.

Recebido para publicação em 03/01/91.
}

rápida degradação em ambiente aberto, em função da maior exposição à luz, a altas temperaturas, à chuva, ao vento, e ocorrência de substratos menos adequados ${ }^{9}$, o peridomicílio é mais precocemente recolonizado por triatomíneos de procedencia silvestre.

Esta experiência foi realizada com o objetivo dese comparar a suscetibilidade do Triatoma sordida à do Triatoma infestans, e de se quantificar a ação residual intradomiciliar da deltametrina e da lambdacyhalotrina nas condições de campo e de trabalho habituais do programa de controle executadio pela SUCAM.

\section{MATERIAL E MÉTODOS}

O trabalho foi realizado no município de Posse-Goiás, nas localidades de Caiçara, Olho DÁgua da Lapa, Pimenta e Formiga, áreas inicialmente com alta infestação pelo $T$. infestanis, e cujas casas apresentavam um padrão razoavelmente homogêneo de construção (pau-a-pique e adobe). As casas que participaram da experiência foram previamente selecionadas, a partir daquelas que constituiam objeto de outra pesquisa (suscetibilidade 
Diotaiuti L, Pinto CT. Suscetibilidade biológica do Triatoma sordida e Triatoma infestans a deltametrina e lambdacyhalotrina em condições de campo. Revista da Sociedade Brasileira de Medicina Tropical 24:151-155, jul-set, 1991

de triatomíneos a vários inseticidas, inclusive os aqui estudados), de responsabilidade da SUCAM. Desta maneira, cada grupo ficou constituído por quatro casas de adobe e uma de pau-a-pique. A partir da avaliação dos seis meses, três destas casas de adobe estavam fechadas, uma em cada grupo, saindo do experimento.

Os produtos utilizados foram deltametrina $5 \%$ nas formulações pó molhável e suspensão concentrada, e lambdacyhalotrina $10 \%$ pó molhável, nas doses pretendidas de $25 \mathrm{mg}$ i.a. $/ \mathrm{m}^{2}$ e $30 \mathrm{mg}$ i.a. $/ \mathrm{m}^{2}$, respectivamente. Não se preocupou com a determinação da dose real aplicada, conforme proposto por Oliveira Filho ${ }^{8}$ em 1988, pois pretendia-se avaliar a ação dos inseticidas obtida rotineiramente pela metodologia da SUCAM. As borrifações foram realizadas em setembro de 1988 pelo pessoal de campo da SUCAM.

A ação inseticida foi medida através de teste de suscetibilidade biológica de triatomíneos, postos em contato por 72 horas a paredes borrifadas. Utilizaram-se, por casa, 10 ninfas de terceiro estádio de $T$. sordida e 10 de $T$. infestans (procedentes do insetário do Laboratório de Biologia de Triatomíneos e Epidemiologia da Doença de Chagas do Centro de Pesquisa René Rachou, Belo Horizonte) em cones plásticos desenvolvidos pela Organização Mundial de Saúde para testes com mosquitos adultos, afixados com pregos e fita adesiva nas paredes. Foram realizadas avaliações aos 90 (dezembro/88), 210 (abril/89), 270 (junho/89) e 360 (setembro) dias após a borrifação. Na última delas, para facilitar a observação do efeito dos inseticidas sobre os insetos, utilizaram-se ninfas de segundo estádio dos triatomíneos. Após 72 horas de contato com os produtos, os cones eram removidos, e os triatomíneos acondicionados em frascos rotulados e encaminhados ao laboratório, onde aos 30 dias, anotava-se o número de mortos. Cada avaliação tinha um grupo controle constituído por $30 \mathrm{~T}$. sordida e $30 T$. infestans, manipulados da mesma forma que os demais insetos (eram levados para o local da experiência e transferidos com pinça para outro recipiente, como se estivessem sendo colocados em cones), exceto pelo fato de não serem expostos a paredes não tratadas (todas as casas rurais da região participaram do teste de suscetibilidade realizado pela SUCAM, estando borrifadas com algum inseticida).

Pretendia-se realizar novos testes até não mais observar-se mortalidade dos insetos; porém, por razões concernentes a própria SUCAM, todas as casas foram novamente borrifadas em setembro de 1989, obrigando-nos a interromper o trabalho.

\section{RESULTADOS}

A Tabela 1 resume os dados obtidos. Observou-se ação inseticida dos três produtos até um ano após a sua aplicação. Do cruzamento dos dados, obteve-se diferença estatisticamente significativa apenas entre a mortalidade de $T$. sordida $(82,5 \%)$ e $T$. infestans $(55 \%)$ expostos a lambdacyhalotrina, aos 360 dias. Aos 210 dias, verificou-se mortalidade semelhante entre 0 controle e os exemplares de $T$. sordida também expostos a paredes borrifadas com este inseticida ( $p>0,05)$. Fora estes resultados que nos chamaram a atenção, a mortalidade obtida com qualquer produto ao longo da experiência foi semelhante. Aos 360 dias, as taxas de mortalidade foram superiores às anteriores.

\section{DISCUSSÃO}

À luz da experiência atual, a eficiência dos piretróides no controle de triatomíneos dispensa maiores comentários. A rápida redução da população doméstica destes insetos após aplicação destes produtos tem sido assinalada desde os primeiros testes realizados com deltametrina no início de década (decamethrin, na época) ${ }^{2}{ }^{10}$, assim como a manutenção das casas livres de triatomíneos por períodos bem maiores que aqueles obtidos pela borrifação com BHC. Por esta última razão, preconizou-se a estratégica utilização de piretróides em áreas já sob controle, não só por sua larga ação residual, mas também por uma esperada ação repelente já identificada sobre mosquitos'. Não se sabe bem se estes inseticidas, a partir de um determinado momento, passam a proteger a casa devido a esta ação repelente e não mais pela ação propriamente inseticida. Neste trabalho foi possível 
Diotaiuti L, Pinto CT. Suscetibilidade biológica do Triatoma sordida e Triatoma infestans a deltametrina $e$ lambdacyhalotrina em condiçóes de cámpo. Revista da Sociedade Brasileira de Medicina Tropical 24:151-155. jul-set, 1991

Tabela 1 - Mortalidade do Triatoma sordida e Triatoma infestans, nos 30 dias após exposição a paredes pulverizadas com deltametrina e lambdacyhalotrina (Municipio de Posse, Estado de Goiás, Brasill 90, 210, 270, e 360 dias após aplicação dos inseticidas.

\begin{tabular}{|c|c|c|c|c|c|c|c|c|}
\hline \multirow{2}{*}{$\begin{array}{l}\text { Produto } \\
\text { formulação }\end{array}$} & \multirow{2}{*}{$\begin{array}{l}\text { Dias após } \\
\text { borrifação }\end{array}$} & \multirow{2}{*}{ Estádio } & \multicolumn{2}{|c|}{ T. sordida } & \multicolumn{2}{|c|}{ T. infestans } & \multicolumn{2}{|c|}{ Total } \\
\hline & & & Expostos & Mortos (\%) & Expostos & Mortos (\%) & Expostos & Mortos (\%) \\
\hline Deltametrina & 90 & $3^{\circ}$ & 44 & $7(15,9)$ & 45 & $15(33,3)$ & 89 & $22(24,7)$ \\
\hline Solução & 210 & $3^{\circ}$ & 40 & $14(35,0)$ & 40 & $21(52,5)$ & 80 & $35(43,8)$ \\
\hline Concentrada & 270 & $3^{\circ}$ & 34 & $11(32,4)$ & 39 & $5(12,8)$ & 73 & $16(21,9)$ \\
\hline $5 \%-25 \mathrm{mg} \mathrm{ia/m^{2 }}$ & 210 & $2^{\circ}$ & 34 & $25(73,5)$ & 35 & $23(65,7)$ & $\circledast 9$ & $48(69,6)$ \\
\hline Deltametrina & 90 & $3^{\circ}$ & 44 & $4(9,1)$ & 43 & $10(23,3)$ & 87 & $14(16,1)$ \\
\hline Pó molhável & 210 & $3^{\circ}$ & 30 & $11(36,7)$ & 30 & $12(40,0)$ & 60 & $23(38,3)$ \\
\hline $5 \%-25 \mathrm{mg} \mathrm{ia} / \mathrm{m}^{2}$ & 270 & $3^{\circ}$ & 30 & $8(26,7)$ & 29 & $4(13,8)$ & 59 & $12(20,3)$ \\
\hline Lambdacyha- & 90 & $3^{\circ}$ & 43 & $12(26,7)$ & 49 & $11(22,4)$ & 94 & $23(24,5)$ \\
\hline lotrina & 210 & $3^{\circ}$ & 30 & $9(30,0)$ & 30 & $16(53,3)$ & 60 & $25(41,7)$ \\
\hline Pó molhável & 270 & $3^{\circ}$ & 39 & $9(23,1)$ & 42 & $9(21,4)$ & 81 & $18(22,2)$ \\
\hline \multirow[t]{2}{*}{$10 \%-30 \mathrm{mg} \mathrm{ia} / \mathrm{m}^{2}$} & 360 & $2^{\circ}$ & 40 & $33(82,5)$ & 40 & $22(55,0)$ & 60 & $55(68,8)$ \\
\hline & 90 & $3^{\circ}$ & 30 & 0 & 30 & 0. & 60 & 0. \\
\hline \multirow[t]{3}{*}{ Controles } & 210 & $3^{\circ}$ & 30 & $2(6,7)$ & 30 & $2(6,7)$ & 60 & $4(6,7)$ \\
\hline & 270 & $3^{\circ}$ & 30 & $2(6,7)$ & 30 & 0 & 60 & $2(3,3)$ \\
\hline & 360 & $2^{\circ}$ & 30 & $7(23,3)$ & 30 & $2(6,7)$ & 6 & $9(15,0)$ \\
\hline
\end{tabular}

observar a ação letal da deltametrina e da lambdacyhalotrina por até 360 dias.

Com qualquer formulação observou-se uma variação nas taxas de mortalidade obtidas, como, por exemplo, com a deltamethrin pó molhável, que na avaliação dos 90 dias promoveu uma mortalidade global dos insetos de $16,1 \%$, e aos 210 dias de $38,3 \%$. Certamente, o local de aplicação dos cones e as possíveis variações na concentração dos inseticidas nas paredes poderiam justificar estes achados. A maior mortalidade com todos os produtos aos 360 dias era esperada, já que foram utilizadas ninfas de segundo estádio, supostamente mais suscetíveis a inseticidas que as demais anteriormente utilizadas.

Em nenhum momento obteve-se uma mortalidade de $100 \%$. As baixas mortalidades obtidas podem ser as características do barro utilizado na confecção do adobe, que podem interferir no potencial inseticida dos produtos testados. Sabe-se que em diferentes tipos de solo, a deltametrina penetra pelo substrato por absorção, processo pelo qual parte do inseticida aspergido se perde'. Por outro lado, o contato com substâncias alcalinas pode degradar mais rapidamente a molécula deste inseticida, reduzindo sua eficiência ${ }^{6}$. No entanto, vale a pena assinalar que as casas que participaram deste teste haviam sido positivas antes da borrifação ( $T$. infestans), e com grande número de triatomíneos por casa, permanecendo negativas um ano após a aspersão dos inseticidas ${ }^{12}$.

Acreditamos que as diferenças observadas entre a mortalidade do $T$. sordida $(82,5 \%)$ e $T$. infestans (55\%) expostos a lambdacyhalotrina aos 360 dias, e entre os exemplares de $T$. sordida expostos a este mesmo inseticida e seus respectivos controles aos 210 dias, ta mbém apresentem caráter circunstancial, relacionados a erros operacionais, 
Diotaiuti L, Pinto CT. Suscetibilidade biologica do Triatoma sordida e Triatoma infestans a deltametrina $e$ lambdacyhalotrina em condições de campo. Revista da Sociedạde Brasileira de Medicina Tropical 24:151-155, jul-set, 1991

na medida em que não se repetiram nas outrás avaliações. Concluímos, portanto, pela igual suscetibilidade do $T$. sordida e $T$. infestans aos produtos testados. Consideramos ser esta um importante informação, já que os resultados das campanhas de borrifação para controle da doença de Chagas nas regiões de ocorrência do $T$. sordida no Brasil ${ }^{5}$ "apontam para indiscutível persistência deste triatomíneo no ambiente peridomiciliar, apesar das borrifações sucessivas com BHC ou peritróides. Esta resistência deve estar relacionaḍa a aspectos compartamentais deste triatomíneo, como sua predominância em montes de lenhâ, galinheiros, paióis, etc, cujas superfícies não podém ser totalmente cobertas com inseticidas, permitindo a permanência de exemplares livres do contato com os mesmos e capazes de se multpilicarem nestes ecótopos, ou mesmo permitindo a sứa reinfestação por exemplares de procedência silvestre ${ }^{4}$.

\section{SUMMARY}

The biological susceptibility of Triatoma sordida and Triatoma infestans to deltame thrin and lambdacyhalothrin was evaluated in three homogeneous groups of houses in the municipality of Posse, Goiás. Each group of houses was treated according to one of the following protocols: deltamethrin $5 \%$ wetted powder at $25 \mathrm{mg}$ a. i. $/ \mathrm{m}^{2}$; deltamethrin $5 \%$ concentrated suspension at $25 \mathrm{mg} \mathrm{a.j.} / \mathrm{m}^{2}$; and lambdacyhalothrin $10 \%$ wetted powder at $30 \mathrm{mg}$ a.i. $/ \mathrm{m}^{2}$. Monitoring of susceptibility to the insecticides was done a fter $90,210,270$ and 360 days after the spraying. Each test consisted of an exposure of 10 third-instar nymphs of each epecies for every house. The insects were kept in contact with the sprayed wall for a period of 72 hours in plastic containers developed by WHO for studies with adult mosquitos. Thirty days after the exposure the death numbers were scored. Results demonstrate persistent activity of the three insecticides for up to a year after spraying and equal biological susceptibilities for both Triatoma sordida and Triatoma infestans to each formulation.

Key-words: Susceptibility, Triatoma sordida. Triatoma infestans. Deltametrina. Lambdacythalotrina.

\section{AGRADECIMENTOS}

Agradecemos ao Dr. Alfredo Martins Oliveira Filho pelas sugestões ao longo do trabalho.
À SUCAM, especialmente ao Dr. João Carlos Pinto Dias, Dr. Isaias Teixeira Neto (DIDOCH, Brasília), e ao pessoal de campo da Diretoria Regional de Goiás, especialmente ao Sr. Lázaro F. Deus, pelo apoio operacional. Ao Centro de Pesquisas René Rachou, pelo fornecimento dos triatomíneos e apoio laboratorial.

\section{REFERÊNCIAS BIBLIOGRÁFICAS}

1. Dartigues V. Utilización de la deltametrina en el control de la malaria. Roussel Uclaf, Division Agrovet, Paris, 1987.

2. Dias JCP, Benedito VA, Vasconcelos JA, Diotaiuti L. Ensaio de campo com permetrina e cipermetrina contra triatomíneos em Minas Gerais, Brasil. In: Resumos do XX Congresso da Sociedade Brasileira de Medicina Tropical, Salvador p.14, 1984.

3. Dias JCP, Pinto CT, Barreiros H, Vasconcelos JRA, Loiola CCP, Silva MA. Emprego da deltametrina e cyflutrina contra triatomíneos em áreas de Minas Gerais, Brasil. Revista da Sociedade Brasileira de Medicina Tropical 19 (supl): 47, 1986.

4. Diotaiuti $L$, Carneiro $M$, Loiola CCP, Silveira Neto HV, Coutinho RM, Dias JCP. Alternativas de controle do Triatoma sordida no Triângulo Mineiro. I - Borrifação parcial (intradomicílio) no município de Douradoquara, MG, Brasil. Revista da Sociedade Brasileira de Medicina Tropical 21; 199-203, 1988.

5. Diotaiuti L, Dias JCP. O peridomicílio no controle vetorial da doença de Chagas, com especial referência ao Triatoma sordida em Minas Gerais. In: Resumos da V Reunião de Pesquisa Aplicada em Doença de Chagas, Araxá p.79, 1988.

6. Fulcones P. Formulación del deltametrin. In: Deltrametrin. Monografia. Roussel Uclaf, Paris p. 139-143, 1983.

7. Lhoste J, L'Hôtellier M. Acciones secundárias del deltametrin sobre el medio ambiente. In: Deltametrin. Monografia. Roussel Uelaf, Paris p.325-357, 1983.

8. Oliveira Filho AM. Development of insecticide formulations and determination of dosages and application schedules to fit specific situations. Revista Argentina de Microbiologia 20 (supl): 3948, 1988 .

9. Oliveira Filho AM. New atternatives for the control 
Diotaiuti L, Pinto CT. Suscetibilidade biológica do Triatoma sordida e Triatoma infestans a deltametrina $e$ lambdacyhalotrina em condiçães de campo. Revista da Sociedade Brasileira de Medicina Tropical 24:151-155, jul-set, 1991

of triatomines in peridomestic buildings. Revista da Sociedade Brasileira de Medicina Tropical 22 (supl II): 53-57, 1989.

10. Pinchin R, Oliveira Filho AM, Fanara DM, Gilbert B. Ensaio de campo para avaliação das possibilidades de uso da decametrina (OMS 1998) no combate a triatomíneos. Revista Brasileira de Malariologia e Doenças Tropicais 32: 36-41 , 1980.
11. Souza AG, Wanderley DV, Buralli GM, Andrade JCR. Consolidation of the control of Chagas disease vectors in the State of São Paulo. Memórias do Instituto Oswaldo Cruz 79 (supl): 125-131, 1984.

12. SUCAM/MS. Relatório referente a ensaios de inseticidas realizados nos municípios de Posse e Galheiros, Divisão de Doença de Chagas/Distrito de Formosa, 1989. 